\title{
ORIGIN OF THE SURFACE SENSITIVITY IN SURFACE X-RAY DIFFRACTION
}

\author{
I. Robinson, P. ENG and R. Schuster \\ Department of Physics, University of Illinois Urbana, IL 61801, USA
}

\begin{abstract}
The first half of this paper explains how X-ray diffraction can be sensitive to surface structure and morphology, even though $X$-rays interact only weakly with matter and hence penetrate deeply into the bulk medium. The basis of the crystal truncation rod construction is given, which demonstrates this sensitivity in a formal way. This is then illustrated with details of two problems of current interest which have been studied with synclırotron radiation at the National Synchrotron Light Source in New York. The structure of the $\mathrm{Si}(111) 7 \times 7$ reconstructed surface, as determined in the vertical direction by $\mathrm{X}$-ray reflectivity, is presented as a straightforward application of crystal truncation rods. Then we discuss alkali adsorption on $\mathrm{Ag}(110)$ surfaces which induces a "missing row" reconstruction. We measured the trends in the induced structural parameters as a function of Cs coverage on $\mathrm{Ag}(110)$, but found the greatest changes were associaled with the location of the Cs instead. At high coverage this is ordered only in a onc-dimensional sense, but as the coverage is reduced it becomes partially registered in the second direction, and, surprisingly, occupies a sitc ligher above the surface.
\end{abstract}

PACS numbers: 07.85.+n, 61.10.Lx

\section{Introduction}

Many people ask why X-ray diffraction, a widely used method of investigating bulk structural properties, should be at all useful for studying surfaces. After all, it is the insensitivity to the poorly controlled surface regions that makes X-ray diffraction so powerful for getting inside to the true bulk of the sample. The response is that synchrotron radiation has changed the world of X-ray diffraction by offering not only unprecedented intensity, but very high reciprocal-space resolution. The overall information content of a diffraction pattern is sufficiently large that it can include details characteristic of both the bulk and surface at the same time. Sensitivity to the bulk arises from simply looking at the bulk part of the pattern; sensitivity to the surface arises from looking at its surface component. This paper will inform the reader how to distinguish one from the other. 


\section{X-ray diffiaction}

The basic principles of $\mathrm{X}$-ray diffraction from surfaces are made simple because the kinematic approximation is generally applicable. For X-ray wavelengths of order $1 \AA$ and relatively light materials the penetration is limited by photoelectric absorption to a depth of microns, provided the grazing angle regime is avoided. Thus, to a reasonable approximation for the near-surface region within about $1000 \AA$ of an accessible surface, there is no penetration problem, and the diffraction from that region can be regarded as superimposed on that of the bulk surrounding it. Needless to say, that bulk contribution is far from negligible as it arises from a far greater amount of material.

An ideal 2D layer, imagined to be somehow floating in space, would have diffraction in the form of continuous lattice rods of constant intensity (ignoring vibration or the finite size of the atoms). If this layer were the surface layer of a crystal, or an interfacial layer, it would have the same periodicity as the bulk of the crystal; the lattice rods would then pass through the discrete Bragg reflections of the bulk. The general construction of the diffraction of a terminated crystal, or one with an internal interface would include the inlerference between these, and there would be a smooth crossover from surface to bulk diffraction. As just described, these lattice rods have the characteristic shape of crystal truncation rods (CTRs) [1]. CTRs are the X-ray equivalent of the continuous rods seen in low energy electron diffraction (LEED), but have orders of magnitude more variation in their intensity because the penetration is so much greater.

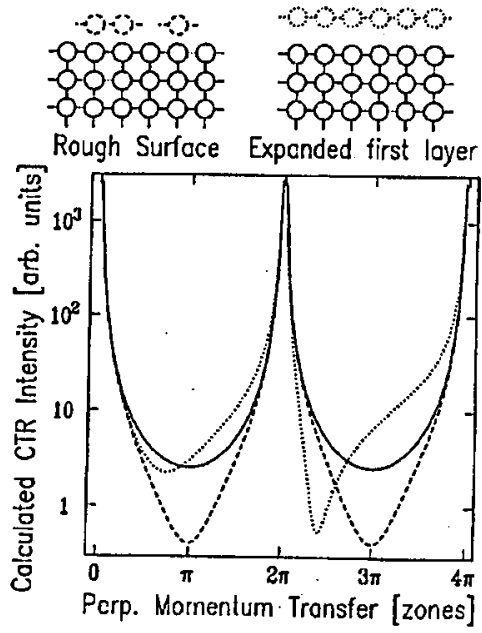

Fig. 1. Theoretical crystal truncation rod (CTR) profiles calculated for three cases: (i) an ideally terminated simply cubic crystal; (ii) the same with top layer roughened by removal of a randomly chosen fraction of atoms; (iii) the same with the top layer spacing enlarged by $10 \%$. For details see text. 
The sensitivity of crystal truncation rods to near-surface region structure and morphology is demonstrated in Fig. 1. Superimposed are calculated curves for an ideally terminated surface, a surface that is rough in the statistical sense of having random atoms omitted in one layer, and a surface with a modification of the spacing. In the vicinity of the divergences of the function (the Bragg points) all curves converge and there is no rcsulting surface structural sensitivity. Elsewhere, and notably at the mid-point of the CTR, there is considerable structural sensitivity, as the different calculated curves demonstrate.

An even more important advantage of X-rays, particularly those produced by electron storage rings at facilities like the ESRF, is thicir very high resolution. The typical resolution function of a 3-axis diffractometer fills only $5 \times 10^{-10}$ of the Brillouin zone of $\mathrm{Si}$. The bulk diffraction is localized in point-like Bragg peaks, smaller than this resolution element. Point defects and bulk thermal diffuse scattering (TDS) are diffuse in all reciprocal space directions. In this way, using symmetry alone, both of these contributions can be eliminated from consideration, leaving only the components of the diffraction with the two-dimensional translational symmetry of surfaces and interfaces which gives rise to the aforementioned rod-like lines of scattering; everything else can be filtered out either by avoidance (3D Bragg peaks) or else by background subtraction (diffuse scattering). Distinguishing among the various interfaces in the problem (for example a buried near-surface region from the surface through which it is measured) is then the only outstanding problem.

\section{X-ray reflectivity study of the $\operatorname{Si}(111)$ surface}

When the bulk crystal has a sufficiently simple structure, in the case of all primitive crystal structures, or the $\mathrm{NaCl}$ structure, there is only one possible way to cut the lattice to reveal a surface. Only in this case, the CTR argument presented above is technically indistinguishable from a simple superposition of $1 / q^{2}$ intensily tails around each Bragg peak [2]. Whenever the crystal has a multiple-atom basis that allows for more than one possible termination, the shape of the CTRs depends on which termination cxists, and is then clearly distinguishable from the superposition of $1 / q^{2}$ intensity tails.

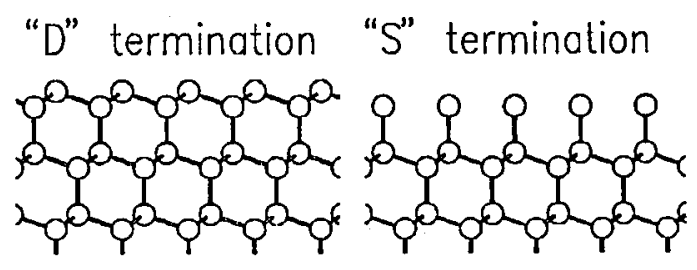

Fig. 2. The two possible terminations of a Si crystal (diamond structure) at its 111 plane.

This is the case of $\mathrm{Si}(111)$, where two possible terminations can exist, as illustrated in Fig. 2. The double-layer (D) tcrmination occurs when bonds perpendicular to the surface are cut; the single-layer $(\mathrm{S})$ termination occurs when we 
break the bonds inclined at $19^{\circ}$ to the surface, as shown. The density of dangling bonds is three times greater for the latter case, so it might be considered less likely to occur. The calculated CIRs are shown for the two cases as lines in Fig. 3. Up to a factor of 10 difference in the two structure factors is scen to occur.

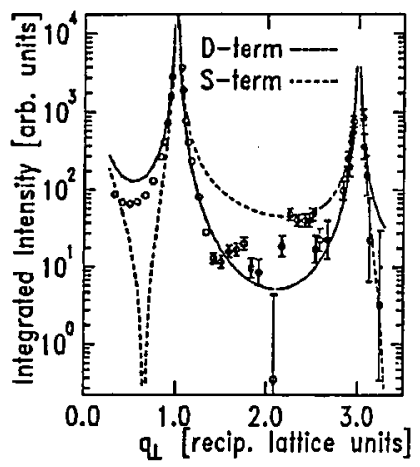

Fig. 3. Measured reflectivity of a UIIV-prepared Si(111) sample, with the theoretical curves for the $\mathrm{S}$ and D terminations supcrimposed (from Ref. [3]).

Also superimposed in Fig. 3 are some carly $X$-ray reflectivity data [3] measured by scanning across the specular C'lR, integrating and subtracting the background intensity. This is the conventional method adopted from X-ray crystallography for measuring reliable structure factors [4]. In reciprocal space, the specular reflectivity line is the direction with the momentum transfer, $q$, entirely perpendicular to the surface. This corresponds exactly to the zero'th order of the CTR diffraction, and so can be thought of as a CTR [1]; the analysis of these data is particularly simple because only the vertical components of the atomic coordinates enter, by virtue of the section-projeclion theorem of Fourier transforms. It is clear that the data fall between the two theoretical curves for the two possible terminations. It is also obvious why this should be the case: the $\operatorname{Si}(111)$ surface when prepared in ultra-high vacuum (UIIV) is not terminated in either simple way; it is reconstructed with $7 \times 7$ translational symmetry.

Figure 4 shows some more recent measurements of the specular CTR of $\mathrm{Si}(111) 7 \times 7$, using a much improved data collection method [5]. A position-sensitive $\mathrm{X}$-ray detector is placed so that it cuts across the difrracted bcam. The background is then measured simultancously with the integrated intensity of the diffracted beam and can be immediately subtracted. The figure also shows the result of a least-squares fit to the data, which passes through the points respectably well. The model used was the dimer-adatom-stacking (DAS) fault model for the Si(111)7 7 reconstructed surface [6]. The heights of the atoms and the vibration amplitudes in the top 4 layers were adjusted for a good fit.

Figure 4 also shows an extra sharp feature in the centre of the scan at the position of the specular 222 Bragg reflection. This is indeed the specular 222 Bragg reflection, which is forbidden in difraction from the ideal diamond lattice, but is 


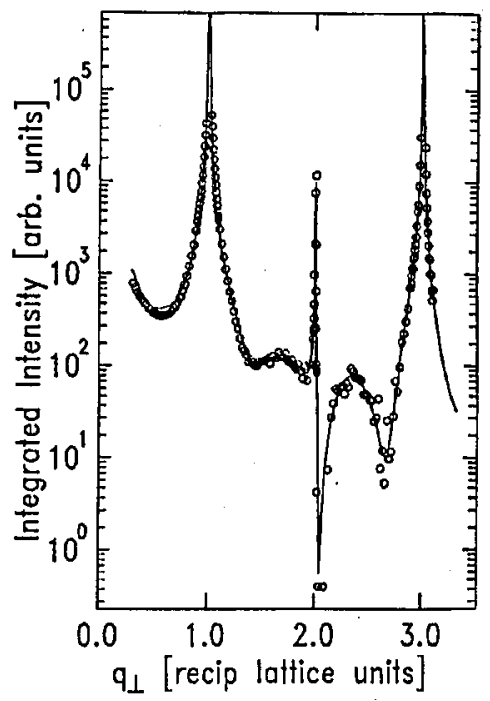

Fig. 4. Improved measurements of the X-ray reflectivity of a UIIV-prepared Si(111) sample, using a position scnsitive detector (Ref. [5]). The solid line is the least squares fit to a model based on the dimer-adatom-stacking-fault (DAS) model for the $7 \times 7$ reconstructed surface.

not perfectly extinguished for two reasons [7]:

(i) The vibrations of the ions in the lattice are not spherically symmetric, but tend to bulge out along the tetrahedral directions (four of the eight $\{111\}$ directions) opposite to each bond; the anisotropy is along different directions for the two atoms in the basis, mcaning they are crystallographically inequivalent.

(ii) The electrons forming the $s p^{3}$-hybridised bonds between the Si atoms are not spherically symmetric either, but have a tetrahedral shape, again meaning the two atoms are crystallographically inequivalent. This affects 4 of the 14 electrons in the $\mathrm{Si}$ form factor so is a relatively large eflect.

It was shown many years ago [7] that contribution (i), which is temperature dependent, is dominant in neutron diffraction, while contribution (ii) is more important in X-ray diffraction experiments. We therefore included extra bond charges in our model to fit this 222 peak in our data. It is orders of magnitude weaker than the bulk diffraction, but still stronger than the surface component of the CTR. A particularly noteworthy feature is the interference betwcen the tails of the 222 and the CTR.

\section{Study of the Cs/Ag(110) surface}

The $\operatorname{Ag}(110)$ surface is of particular interest because of its marginal stability when it is clean. The (110) surface of the analogous third row transition element, $\mathrm{Au}$, is reconstructed with the well-known $1 \times 2$ "missing row" structure. Clean 
$\mathrm{Ag}(110)$ is unreconstructed, but as theory has shown [8], the energy difference between the unreconstructed and the reconstructed is close to zero. Experimentally, it is found that a moderate amount of alkali metal deposited on the clean surface transforms it from unreconstructed to $1 \times 2$ reconstructed [9]. The appropriate coverage range for the reconstruction, in the case of Cs adsorption, was found to be around $\Theta_{\mathrm{Cs}}=0.15$ to $\Theta_{\mathrm{Cs}}=0.3$ [9]. Since these effects can be considered in a general sense to be due to an alkali-induced alteration of the surface free energy via the chemical potential, we were interested in whether there was any apparent structural response to this change within the $\Theta_{\mathrm{Cs}}=0.15$ to $\Theta_{\mathrm{Cs}}=0.3$ range over which the surface has the $1 \times 2$ reconstruction.

We therefore measured the surface diffraction intensity distribution for two values of coverage that were at the extreme ends of the range of ordered $1 \times 2$ structures (as determined by a well-ordered diffraction pattern). In our experiment this criterion was found to give $\Theta_{\mathrm{Cs}}=0.2$ and $\Theta_{\mathrm{Cs}}=0.3$, as determined by Auger spectroscopy, so these were the coverages we examined [10]. The data are shown in Figs. 5 and 6. Careful comparison shows there are indeed differences between the structures at the two coverages.

The subsequent structure factor analysis of these data led to a complete surprise, however. We had expected to see systematic changes in the pattern of subsurface relaxations that accompanies the basic missing row structure; instead we found that these were small, but not dramatic, and certainly did not explain the observed changes in the data. Conversely, we had not expected to see ordered Cs atoms in the structure, since previous LEED studies of this surface [11] had not done so; instead we found clear evidence for ordered Cs. This can be seen most directly in the $(0.5,0, L)$ data of Fig. 5 , which we now discuss: at this position the total parallel momentum transfer is very small, so the effects of relaxations (displacements) should be almost invisible. In this approximation, a missing row structure would then appear to contain a single $\mathrm{Ag}$ atom per unit cell and nothing else, leading to a flat $(0.5,0, L)$ rod with no intensity variation along it. The fact that we do observe a sinusoidal variation can only be attributed to a contribution from the Cs adsorbed layer interfering with that from the single $\mathrm{Ag}$ atom. The period of this intensity modulation is the inverse of the distance between the layers. Thus we see ordered Cs in the structure, and, as Fig. 5 shows, its distance above the top Ag layer varies with coverage.

The direction of this trend is clear: higher $\Theta_{\mathrm{Cs}}$ gives a less rapid modulation in reciprocal space, implying a shorter distance in real-space. This is borne out by a complete structure factor analysis of all the data [10]. The result is, however, contrary to conventional wisdom concerning the forces involved. A number of related theoretical arguments all lead to the same conclusion: whenever more alkali atoms are packed together, the bonding within the layer gets stronger and the bonding to the substrate therefore gets weaker. It is normally expected then that the distance between the alkali layer and the substrate should increase with coverage.

While we cannot at present explain why this happens, the careful analysis of the data in Figs. 5 and 6 does allow us to elaborate on the question of how it happens. To obtain the best fit to all the data, and particularly the $(0.5,1, L)$ data, 


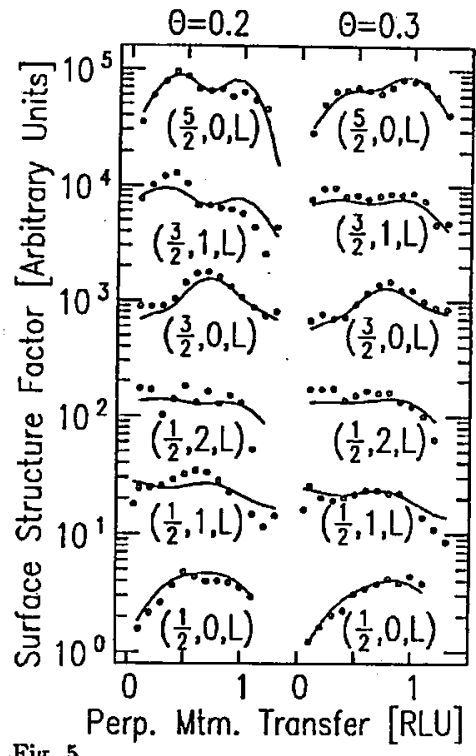
Fig. 5

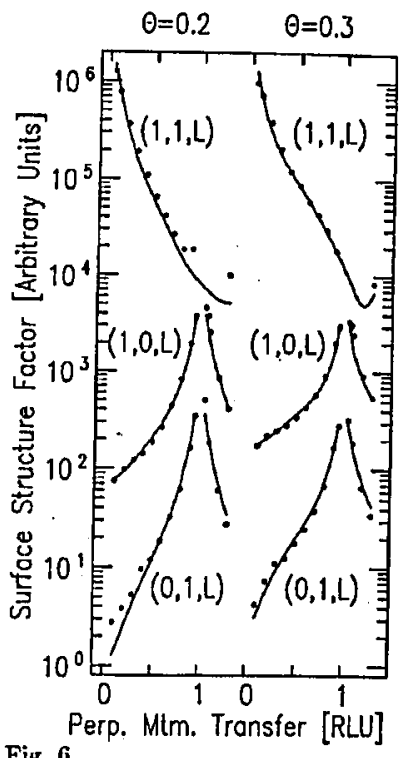

Fig. 6

Fig. 5. Fractional order structure factor data measured for $\mathrm{Cs} / \mathrm{Ag}(110)$ at coverages $\Theta_{\mathrm{Cs}}=0.2$ and $\Theta_{\mathrm{Cs}}=0.3$. The square root of the intensity at reciprocal space position $(H, K, L)$ is plotted vs. $L$ for different $H$ and $K$ for half-order values of $H$. The curves have been offset for clarity. These contributions arise exclusively from the surface region. Fig. 6. Integer order structure factor data measured for $\mathrm{Cs} / \mathrm{Ag}(110)$ at coverages $\Theta_{\mathrm{Cs}}=0.2$ and $\Theta_{\mathrm{Cs}}=\mathbf{0 . 3}$. The square root of the intensity at reciprocal space position $(I, K, L)$ is plotted vs. $L$ for different $I I$ and $K$ for integer values of $H$. The curves have been offset for clarity. The divergence at certain discrete $L_{\mathrm{Bragg}}$ valucs corresponds to a bulk Bragg peak, and the asymptotic region is the crystal truncation rod that becomes progressively more surface sensitive as $L$ departs from $L_{\mathrm{Bragg}}$.

it is important to address the question of how the Cs atoms are distributed along the troughs of the missing row structure. A good fit to $\Theta_{\mathrm{Cs}}=0.3$ and a reasonable fit to $\Theta_{\mathrm{Cs}}=0.2$ resulted from the simple assumption that the Cs was completely disordered in that direction, so only contributed to data with indices $(H, 0, L)$ and not at all to $(H, K, L)$ with $K \neq 0$. An improved fit to $\Theta_{\mathrm{Cs}}=0.2$ came from relaxing the assumptions slightly by allowing a fraction of the Cs atoms to sit in ordered 4-fold sites. If we then make the assumption that surface is phase separated into low-coverage regions in 4-fold sites (large height) and higher coverage regions with incommensurate sites (smaller height), we can explain the observed coverage dependence as a kind of condensation. As far as we can tell, the only requirement for this scenario would be an interaction potential between $\mathrm{Cs}$ atoms bound on the substrate which is repulsive at large distance and attractive at smaller distances, i.e. there is a potential barrier between two bound adsorbate atoms. There is some theoretical justification for this possibility [12]. 


\section{Acknowledgments}

E. Vlieg made important contributions to the earlier reflectivity measurements on Si(111) in Fig. 3. Support for this work came from the University of Illinois Materials Research Laboratory under grant DEFG02-91ER45439. NSLS is supported by the U.S. Department of Energy under grant DE-AC012-76CII00016.

\section{References}

[1] I.K. Robinson, Phys: Rev. B 33, 3830 (1986).

[2] I.K. Robinson, Faraday Discuss. R. Soc. Chem. 80, 208 (1990).

[3] I.K. Robinson, E. Vlieg, Surf. Sci. 261, 123 (1992).

[4] I.K. Robinson, in: IIandbook on Synchrotron Radiation, Eds. D.E. Moncton, G.S. Brown, Vol. 3, Elsevier, North-IIolland, Amsterdam 1990.

[5] I.K. Robinson, P.J. Eng, to be published.

[6] K. Takayanagi, Y. Tanishiro, S. Takahiashi, M. Takahtaslii, Surf. Sci. 164, 367 (1985).

[7] D. Keating, A. Nunes, B. Batterman, J. Ifastings, Phys. Rev. B 4, 2472 (1971).

[8] K.M. Iо, K.P. Bohnen, Europhys. Lett. 4, 345 (1987); K.M. IIo, K.P. Bohnen, Phys. Rev. Lelt. 59, 1833 (1987).

[9] M. Lindroos, C.J. Barnes, M. Valden, D.A. King, Surf. Sci. 218, 269 (1989); B.E. IIayden, K.C. Prince, P.J. Davie, G. Paolucci, A.M. Bradshaw, Solid Slate Commun. 48, 325 (1983); R. Döll-Oclze, E.M. Stuve, J.K. Sass, Solid State Commun. 57, 323 (1986); J.W.M. Frenken, R.L. Krans, J.F. van der Veen, E. Holub-Krappe, K. IIorn, Phys. Rev. Lelt. 59, 2307 (1987).

[10] R. Schuster, P.J. Eng, I.K. Robinson, to be published.

[11] H. Over, II. Bludau, M. Skottkc-Kílein, G. Ertl, W. Moritz, C.T. Campbell, Phys. Rev. B 45, 8638 (1992).

[12] J. Neugebauer, M. Schefller, Phys. Rev. Lelt. 71, 577 (1993). 